\title{
Unusual Case of an Intracranial Aneurysm Misdiagnosed as Focal Basal Meningitis
}

*Philips G. Michael, Rakesh M. Jamkhandikar, Imran K. Memon, Tahra Al-Saadi, Said Al-Aghbari, Majed Al-Muqaimi, Misbahuddin Ansari, Ahmed Al-Shamakhi, Asifa Ahmad
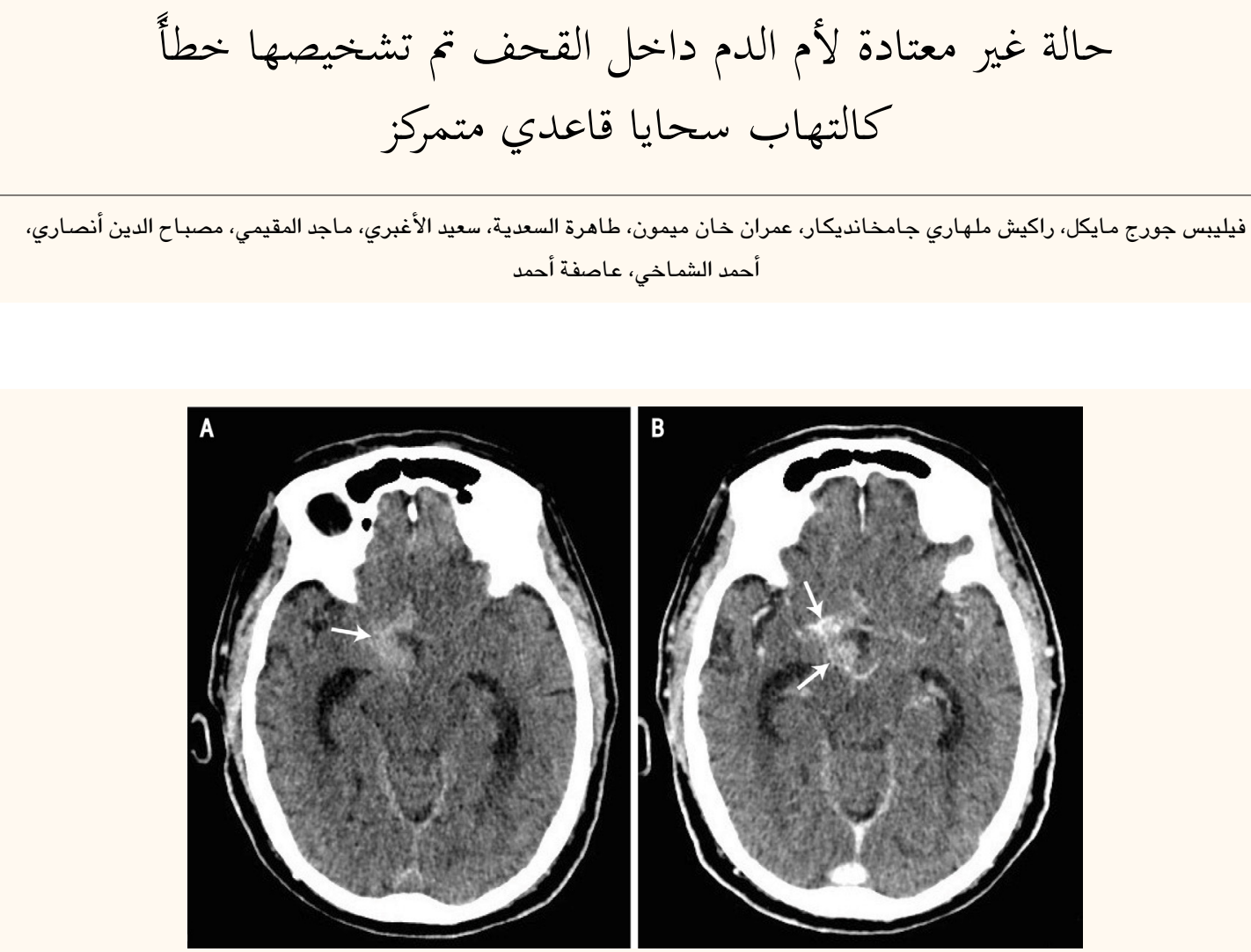

Figure 1: Initial (A) pre- and (B) post-contrast axial computed tomography scans of the brain of a 67-year-old male patient showing an irregular curvilinear and nodular area of abnormal enhancement on the right side of the prepontine and suprasellar cistern extending to the pituitary fossa (arrows). This was initially diagnosed as focal basal meningitis.

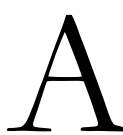

67-YEAR-OLD DIABETIC MALE PATIENT PRESented to the Accident \& Emergency Department of the Armed Forces Hospital, Muscat, Oman, in 2016 with a headache, low-grade intermittent fever and progressive confusion with decreased sensorium of two weeks' duration. The patient had a past history of malignant otitis externa along with right-sided mastoiditis for which he had undergone a cortical mastoidectomy and debridement seven months previously. He had subsequently developed right-sided facial palsy immediately after the surgery. The patient also disclosed a recent history of admission to a local secondary hospital, at which time a computed tomography (CT) scan of the brain was reportedly unremarkable. An unsuccessful lumbar puncture was attempted and, shortly afterwards, the patient had left the previous hospital against medical advice at the behest of his relatives and sought admission to the Armed Forces Hospital. Unfortunately, no detailed documentation, prior medical reports or imaging scans were available since the patient had been discharged without following the proper referral procedures.

At admission, the patient was clinically afebrile upon examination; however, there was evidence of neck rigidity for which a contrast-enhanced CT scan of the brain was requested to rule out meningitis. The CT scan showed postoperative changes of right-sided otomastoiditis with chronic osteomyelitis. Apart from this, there was an irregular curvilinear and nodular area of abnormal enhancement on the right side of the prepontine and suprasellar cistern extending to the pituitary fossa which was deemed indicative of focal 


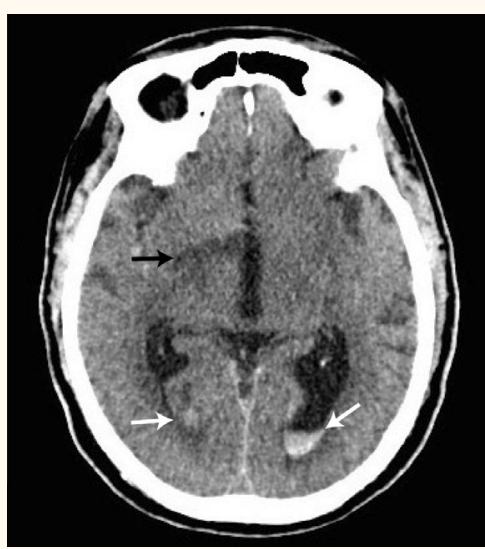

Figure 2: A follow-up non-contrast axial computed tomography scan of the brain of a 67-year-old male patient initially diagnosed with focal basal meningitis showing intraventricular haemorrhage in the dependent part of both lateral ventricles (white arrows), with a developing hypodense area of infarction in the region of the right middle cerebral artery (black arrow).

basal meningitis [Figure 1]. In view of these findings, a repeat lumbar puncture was planned; however, the patient's relatives were initially unwilling to consent to the procedure. As such, the lumbar puncture was delayed by 24 hours and, when attempted, was traumatic and unsuccessful. Subsequently, the patient was prescribed intravenous antibiotics but failed to improve. In view of the lack of response to treatment and the patient's impaired renal function, repeat noncontrast CT imaging of the brain was performed 48 hours after the initial scan. This demonstrated minimal intraventricular haemorrhage in the dependent part of both lateral ventricles, with a developing area of infarction in the region of the right middle cerebral artery (MCA) [Figure 2]. Apart from this, there were no significant changes in comparison to the initial CT scan.

Over the next three days, the patient's condition further deteriorated and he was transferred to the Intensive Care Unit where he required intubation and ventilation. Five days after admission, another follow-up contrast-enhanced CT scan demonstrated a leaking aneurysm arising from the right MCA with subarachnoid and intraventricular haemorrhage [Figure 3]. Upon review of the initial CT images, it was revealed that the abnormality initially considered to indicate focal basal meningitis was actually an MCA aneurysm. As there were no neurosurgical and vascular interventional departments available at the Armed Forces Hospital, an urgent consultation was sought with neurosurgical and interventional neuroradiology experts at a nearby institution; unfortunately, the patient could not be immediately referred as his condition was very unstable and he died the following day.

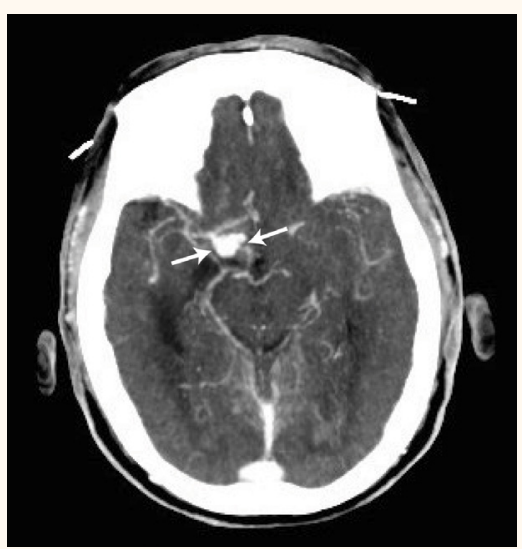

Figure 3: A final axial post-contrast computed tomography image of the brain of a 67-year-old male patient initially diagnosed with focal basal meningitis showing a leaking aneurysm in the right middle cerebral artery (arrow).

\section{Comment}

Despite significant improvements in imaging technology, diagnostic radiological errors can still occur due to poor imaging techniques or failure of perception, lack of knowledge or misjudgment on the part of the radiologist. ${ }^{1}$ As such, both diagnostic radiologists and referring physicians should be aware of potential sources of error when interpreting imaging scans. Errors in which an abnormality is detected on imaging but its true pathological nature and significance are not recognised are classified as cognitive errors; these include false-positive cases as well as the inaccurate classification of abnormal findings. ${ }^{1}$ Erroneous conclusions drawn from improper clinical information, a paucity of knowledge or the consideration of only a restricted number of differential diagnoses have been previously implicated in misdiagnoses. ${ }^{1}$

In the current case, although the abnormality was correctly identified on the initial CT scan, it was falsely attributed to focal basal meningitis rather than a ruptured aneurysm; this represents a rare instance of cognitive error. In hindsight, magnetic resonance imaging (MRI) might have helped to detect the haemorrhage and ensured that the correct diagnosis was made at an earlier stage. However, the patient's condition was not stable enough for an emergency MRI at presentation; moreover, the Armed Forced Hospital did not have MRI-compatible monitoring devices for such patients at the time. In addition, the patient's previous history of otitis externa, mastoiditis and surgery as well as the clinical suspicion of meningitis for which the scan was requested may also have played a role in misleading the radiologist, resulting in an erroneous diagnosis. When interpreting imaging findings, care must be taken so as not to become overdependent on the patient's medical history 
and clinical information as this could be a source of potential bias. It is vital that radiologists resist the temptation to fit imaging findings with the clinical diagnosis.

Malatt et al. identified several factors contributing to false-negative interpretations of emergency head CT scans, including inadequate or inaccurate clinical information, failure to compare recent images with those from prior scans, use of improper windowing and leveling settings and lack of use of multiplanar reconstruction technology to provide various anatomical views. ${ }^{2}$ Lawson et al. also reported that excessive reliance on previous experience, the inclination to find and/or exaggerate supporting evidence to favour a specific diagnosis while neglecting or failing to obtain opposing evidence and the penchant to make a diagnosis without sufficient testing could all contribute to bias and result in an erroneous diagnosis. ${ }^{3}$ It is therefore essential to keep an open mind and consider relevant alternative diagnoses when interpreting a specific imaging finding.

Intravenous contrast $\mathrm{CT}$ imaging and optimal contrast bolus timing may also play a crucial role in establishing the diagnosis of an intracranial aneurysm. ${ }^{4}$ In the present case, the final CT scan was in the angiographic (i.e. arterial) phase and unequivocally established the presence of an MCA aneurysm.
However, the initial post-contrast CT scan was not performed in the angiographic phase; it is therefore possible that, had the initial images been obtained in the optimal arterial phase, the correct diagnosis could have been established earlier. Taheri et al. have previously highlighted unusual configurations and presentations of intracranial aneurysms which may mimic neoplastic lesions or an infectious process and therefore be misdiagnosed on imaging. ${ }^{5}$

\section{References}

1. Bahrami S, Yim CM. Quality initiatives: Blind spots at brain imaging. Radiographics 2009; 29:1877-96. doi: 10.1148/rg.2 97095123.

2. Malatt C, Zawaideh M, Chao C, Hesselink JR, Lee RR, Chen JY. Head computed tomography in the emergency department: A collection of easily missed findings that are lifethreatening or life-changing. J Emerg Med 2014; 47:646-59. doi: 10.1016/j.jemermed.2014.06.042.

3. Lawson AE, Daniel ES. Inferences of clinical diagnostic reasoning and diagnostic error. J Biomed Inform 2011; 44:402-12. doi: 10.1016/j.jbi.2010.01.003.

4. Yoon NK, McNally S, Taussky P, Park MS. Imaging of cerebral aneurysms: A clinical perspective. Neurovasc Imaging 2016; 2:6-13. doi: 10.1186/s40809-016-0016-3.

5. Taheri SM, Haghighatkhah HR, Noori M. Uncommon patterns of intracranial aneurysms: A pictorial essay. Iran J Radiol 2009; 6:237-46. 